\title{
Tripartite Entanglement-Dependence of Tripartite Non-locality in Non-inertial Frame
}

\author{
DaeKil Park ${ }^{1,2}$ \\ ${ }^{1}$ Department of Physics, Kyungnam University, Changwon, 631-701, Korea \\ 2 Department of Electronic Engineering, \\ Kyungnam University, Changwon, 631-701, Korea
}

\begin{abstract}
The three-tangle-dependence of $S_{\max }=\max \langle S\rangle$, where $S$ is Svetlichny operator, are explicitly derived when one party moves with an uniform acceleration with respect to other parties in the generalized Greenberger-Horne-Zeilinger and maximally slice states. The $\pi$-tangle-dependence of $S_{\max }$ are also derived implicitly. From the dependence we conjecture that the multipartite entanglement is not the only physical resource for quantum mechanical multipartite non-locality.
\end{abstract}


After Einstein-Podolsky-Rosen's seminal paper [1] the unusual properties of the quantum correlations became a fundamental issue in quantum information theories. This unusual properties become manifest if one examines Bell inequality $\langle\mathcal{B}\rangle \leq 2[2]$ by making use of bipartite quantum states. If this inequality is violated, this fact guarantees the nonlocality of quantum mechanics. As Gisin[3] showed, the Bell-type Clauser-Horner-ShimonyHolt (CHSH) [4] inequality is violated for all pure entangled two-qubit states. This fact implies that quantum mechanics really exhibits non-local correlations. More importantly, the amount of violation $\langle\mathcal{B}\rangle-2$ increases when the two-qubit state is entangled more and more. This fact implies that the origin of the non-local correlations in quantum mechanics is an entanglement of quantum states. This remarkable fact can be used to implement the quantum cryptography[5].

Although the relationship between non-locality and entanglement is manifest to a great extent in two-qubit system, it is not straightforward to explore this relationship in multipartite system. Recently, however, understanding in this direction is enhanced little bit, especially in three-qubit system. In Ref. [6] the relationship between Svetlichny inequality[7], the Bell-type inequality in tripartite system, and tripartite residual entanglement called three-tangle [8] was examined by making use of the generalized Greenberger-Horne-Zeilinger (GHZ) states $\left|\psi_{g}\right\rangle[9]$ and the maximally slice (MS) states $\left|\psi_{s}\right\rangle$ [10] defined as

$$
\begin{aligned}
& \left|\psi_{g}\right\rangle=\cos \theta_{1}|000\rangle+\sin \theta_{1}|111\rangle \\
& \left|\psi_{s}\right\rangle=\frac{1}{\sqrt{2}}\left[|000\rangle+|11\rangle\left\{\cos \theta_{3}|0\rangle+\sin \theta_{3}|1\rangle\right\}\right] .
\end{aligned}
$$

The most remarkable fact Re.[6] found is that the $\tau$ (three-tangle)-dependence of $S_{\max }$, the upper bound of expectation value of the Svetlichny operator, for $\left|\psi_{g}\right\rangle$ is

$$
S_{\text {max }}\left(\psi_{g}\right)=\left\{\begin{array}{cc}
4 \sqrt{1-\tau} & \tau \leq 1 / 3 \\
4 \sqrt{2 \tau} & \tau \geq 1 / 3 .
\end{array}\right.
$$

Since the Svetlichny inequality is $\langle S\rangle \leq 4$, whose violation guarantees the non-local correlations, Eq. (2) shows that $\left|\psi_{g}\right\rangle$ really exhibits non-local correlations in the region $\tau>1 / 2$. Unlike two-qubit states, however, $S_{\max }$ exhibits a decreasing behavior when $\tau \leq 1 / 3$. This fact strongly suggests that the quantum entanglement is not the only resource for the multipartite non-locality. It seems to be greatly important issue to find the other resources, which are responsible for the non-local properties of quantum mechanics. 
The purpose of this paper is to examine the relationship between tripartite entanglement and $S_{\max }$ in non-inertial frames. Since entanglement and non-locality are the two most important concepts in quantum mechanics, the relationship between them is at the heart of the foundations of quantum mechanics. Recently, the relations for several 3-qubit pure states were derived in the non-relativistic framework [6, 11]. The original purpose of this paper is to extend these relations to the relativistic framework. Since, furthermore, the analysis in the non-inertial frames generally transforms a pure state into a mixed state due to Unruh decoherence effect [12, 13], as a by-product one can derive the relationship between tripartite entanglement and $S_{\max }$ for various mixed states in this paper.

Although similar issue was considered recently in Ref. [14], authors in this reference chose only $\pi$-tangle [15] as a tripartite entanglement measure. However, the explicit $\pi$-tangledependence of $S_{\max }$ was not derived in Ref.[14]. As far as we know, furthermore, there are two different tripartite entanglement measures such as three-tangle[8] and $\pi$-tangle[15]. Unlike $\pi$-tangle, three-tangle has its own historical background. In fact, it exactly coincides with the modulus of a Cayley's hyperdeterminant[16, 17], which was constructed long ago. It is also polynomial invariant under the local $S L(2, \mathbb{C})$ transformation[18, 19]. Thus, it seems to be more meaningful to derive the three-tangle-dependence of $S_{\max }$ explicitly.

However, the calculation of three-tangle for three-qubit mixed states is much more difficult than that of $\pi$-tangle. Since three-tangle for mixed state $\rho$ is defined by convex roof $\operatorname{method}[20,21]$

$$
\tau(\rho)=\min \sum_{j} P_{j} \tau\left(\rho_{j}\right),
$$

where minimum is taken over all possible ensembles of pure states $\rho_{j}$ with $0 \leq P_{j} \leq 1$, the explicit computation of three-tangle needs to derive an optimal decomposition of the given mixed state $\rho$. It causes difficulties in the analytic computation of the three-tangle. Recently, however, various techniques [22 27] were developed to overcome these difficulties. Still, however, it is highly non-trivial task to compute the three-tangle analytically for highrank mixed states except very rare cases. Fortunately, the mixture derived in this paper is only rank-two. Thus, it is possible to compute the three-tangle analytically using various techniques developed in Ref. [22 27]. In this paper we use these techniques to derive the relations between the three-tangle and $S_{\max }$ in non-inertial frames.

Now, we assume that Alice, Bob, and Charlie initially share the generalized fermionic 
GHZ state $\left|\psi_{g}\right\rangle_{A B C}$ or the MS state $\left|\psi_{s}\right\rangle_{A B C}$. We also assume that after sharing his own qubit, Charlie moves with respect to Alice and Bob with a uniform acceleration $a$. Then, Charlie's vacuum and one-particle states $|0\rangle_{M}$ and $|1\rangle_{M}$, where the subscript $M$ stands for Minkowski, are transformed into[28]

$$
\begin{aligned}
|0\rangle_{M} & \rightarrow \cos r|0\rangle_{I}|0\rangle_{I I}+\sin r|1\rangle_{I}|1\rangle_{I I} \\
|1\rangle_{M} & \rightarrow|1\rangle_{I}|0\rangle_{I I}
\end{aligned}
$$

where the parameter $r$ is defined by

$$
\cos r=\frac{1}{\sqrt{1+\exp (-2 \pi \omega c / a)}},
$$

and $c$ is the speed of light, and $\omega$ is the central frequency of the fermion wave packet ${ }^{1}$. Thus, $r=0$ when $a=0$ and $r=\pi / 4$ when $a=\infty$. In Eq. (44) $|n\rangle_{I}$ and $|n\rangle_{I I}(n=0,1)$ are the mode decomposition in the two causally disconnected regions in Rindler space. Therefore, Eq. (4) implies that the physical information initially formed in region $I$ is leaked into the region $I I$, which is a main story of the Unruh effect [12, 13].

Before we discuss on the relationship between Svetlichny inequality and tripartite entanglement, we should comment that the superselection rule (SSR) of the fermion fields[29] does not allow $\left|\psi_{g}\right\rangle_{A B C}$ and $\left|\psi_{s}\right\rangle_{A B C}$ as fermion states. This can be easily confirmed by the fact that $\left|\psi_{g}\right\rangle\left\langle\psi_{g}\right|$ and $\left|\psi_{s}\right\rangle\left\langle\psi_{s}\right|$ are not commute with $(-1)^{\hat{F}}=\operatorname{diag}\{1,-1,-1,1,-1,1,1,-1\}$, where $\hat{F}$ is the fermion number operator [30]. Recently, the SSR and some other subtle issues for the fermion fields were discussed in the context of the relativistic quantum information theories [31 33]. Furthermore, as discussed in Ref.[30], this SSR constraint also modifies the definition of the three-tangle for the mixed states because the optimal decompositions also should obey the SSR constraint. If, therefore, the SSR is taken into account, Eq. (3) yields merely the lower bound of the three-tangle.

${ }^{1}$ For bosonic state Eq.(4) is changed into

$$
|0\rangle_{M} \rightarrow \frac{1}{\cosh r} \sum_{n=0}^{\infty} \tanh ^{n} r|n\rangle_{I}|n\rangle_{I I} \quad|1\rangle_{M} \rightarrow \frac{1}{\cosh ^{2} r} \sum_{n=0}^{\infty} \tanh ^{n} r \sqrt{n+1}|n+1\rangle_{I}|n\rangle_{I I},
$$

where

$$
\cosh r=\frac{1}{\sqrt{1-\exp (-2 \pi \omega c / a)}}
$$


In spite of this fact we will neglect the restriction generated by SSR in this paper. The main reason for this is that as Weinberg commented in Ref. [29], it is always possible to enlarge the symmetry group to a new one that lacks the SSR. Thus, it is possible to remove the SSR restriction by extending the symmetry group appropriately.

Using Eq. (4) one can easily show that the Charlie's acceleration makes $|\psi\rangle_{A B C}$ to be

$$
|\psi\rangle_{A B C} \rightarrow\left[\cos \theta_{1} \cos r|000\rangle+\sin \theta_{1}|111\rangle\right] \otimes|0\rangle_{I I}+\cos \theta_{1} \sin r|001\rangle \otimes|1\rangle_{I I}
$$

where $|\alpha \beta \gamma\rangle \equiv|\alpha \beta\rangle_{A B}^{M} \otimes|\gamma\rangle_{I}$. Since $|\psi\rangle_{I I}$ is a physically inaccessible state from region $I$, it is reasonable to take a partial trace over $I I$ to average it out. Then, the remaining quantum state becomes the following mixed state:

$$
\begin{aligned}
\rho_{A B I} & =\cos ^{2} \theta_{1} \cos ^{2} r|000\rangle\left\langle 000\left|+\cos ^{2} \theta_{1} \sin ^{2} r\right| 001\right\rangle\left\langle 001\left|+\sin ^{2} \theta_{1}\right| 111\right\rangle\langle 111| \\
& +\sin \theta_{1} \cos \theta_{1} \cos r\{|000\rangle\langle 111|+| 111\rangle\langle 000|\} .
\end{aligned}
$$

The maximum of the expectation value of the Svetlichny operator, $S_{\max }$, for $\rho_{A B I}$ was explicitly derived in Ref.[14], and the final expression can be written as

$$
S_{\text {max }}=4 \max \left[\left|2 \cos ^{2} \theta_{1} \cos ^{2} r-1\right|, \sqrt{2}\left|\sin 2 \theta_{1}\right| \cos r\right] .
$$

When $a=0$, Eq. (8) reduces to $S_{\max }=4 \max \left[\left|2 \cos ^{2} \theta_{1}-1\right|, \sqrt{2}\left|\sin 2 \theta_{1}\right|\right]$, which ensures that the violation of the Svetlichny inequality arises when $\pi / 8<\theta_{1}<3 \pi / 8$ in a region $0 \leq \theta_{1} \leq \pi / 2$. When $a=\infty$, Eq. (8) reduces to $S_{\max }=4 \max \left[1-\cos ^{2} \theta_{1}, \sin 2 \theta_{1}\right]$, which shows that there is no violation of the Svetlichny inequality.

Now, we discuss on the tripartite entanglement of $\rho_{A B I}$ given in Eq. (17). The computation of its $\pi$-tangle is straightforward and the final expression becomes

$$
\pi_{G G H Z}=\frac{2+\cos ^{2} r}{3} \sin ^{2} 2 \theta_{1}+\frac{1}{3} \cos ^{4} \theta_{1} \sin ^{2} 2 r .
$$

When, therefore, $a=0, \pi_{G G H Z}$ becomes $\sin ^{2} 2 \theta_{1}$, which shows that $\left|\psi_{g}\right\rangle$ is maximally entangled at $\theta_{1}=\pi / 4$ and non-entangled at $\theta_{1}=0$ and $\pi / 2$. When $a=\infty$, Eq. (9) reduces to $\pi_{G G H Z}=(5 / 6) \sin ^{2} 2 \theta_{1}+(1 / 3) \cos ^{4} \theta_{1}$, which is maximized by $25 / 27 \sim 0.926$ at $\theta_{1}=\sin ^{-1}(2 / 3)$ and minimized by zero at $\theta_{1}=\pi / 2$. The nonvanishing tripartite entanglement at $a \rightarrow \infty$ limit was discussed in Ref.[34]. This property is a crucial difference from the bosonic bipartite entanglement, which completely vanishes at $a \rightarrow \infty$ limit [35]. 
In order to compute the three-tangle it is convenient to use the spectral decomposition of $\rho_{A B I}$, whose expression is

$$
\rho_{A B I}=p|G H Z\rangle\langle G H Z|+(1-p)| 001\rangle\langle 001|,
$$

where $|G H Z\rangle=a|000\rangle+b|111\rangle$ with

$$
p=\cos ^{2} \theta_{1} \cos ^{2} r+\sin ^{2} \theta_{1} \quad a=\frac{\cos \theta_{1} \cos r}{\sqrt{\sin ^{2} \theta_{1}+\cos ^{2} \theta_{1} \cos ^{2} r}} \quad b=\frac{\sin \theta_{1}}{\sqrt{\sin ^{2} \theta_{1}+\cos ^{2} \theta_{1} \cos ^{2} r}} .
$$

In order to derive the optimal decomposition we define

$$
|Z(\phi)\rangle=\sqrt{p}|G H Z\rangle+e^{i \phi} \sqrt{1-p}|001\rangle
$$

This has several interesting properties. First, $\rho_{A B I}$ given in Eq.(10) can be written as

$$
\rho_{A B I}=\frac{1}{2}[|Z(\phi)\rangle\langle Z(\phi)|+| Z(\phi+\pi)\rangle\langle Z(\phi+\pi)|] .
$$

Second, the three-tangle of $|Z(\phi)\rangle$ is independent of $\phi$ as $\tau_{Z}=4 p^{2} a^{2} b^{2}$. If, therefore, Eq. (13) is an optimal decomposition, the three-tangle of $\rho_{A B I}$ is also $\tau_{A B I}=4 p^{2} a^{2} b^{2}$. Since $\tau_{A B I}$ is convex with respect to $p$, this fact guarantees that Eq. (13) is really optimal decomposition for $\rho_{A B I}$. Using Eq. (11) it is easy to show

$$
\tau_{A B I}=\sin ^{2} 2 \theta_{1} \cos ^{2} r
$$

Therefore, combining Eq. (8) and Eq. (14) we get the explicit three-tangle-dependence of $S_{\max }$ as following;

$$
S_{\text {max }}=4 \max \left[\sqrt{\cos ^{2} r-\tau_{A B I}} \cos r-\sin ^{2} r, \sqrt{2 \tau_{A B I}}\right] .
$$

When $a=0$, it is easy to to show that Eq. (2) is reproduced.

\begin{tabular}{c||c|c|c|c|c|c|c|c}
\hline$a / \omega c$ & 0 & 2 & 4 & 6 & 8 & 10 & 100 & $\infty$ \\
\hline \hline$\pi_{*}$ & 0.50 & 0.563 & 0.70 & 0.757 & 0.787 & 0.806 & 0.901 & 1 \\
\hline$\tau_{*}$ & 1 & 0.959 & 0.828 & 0.740 & 0.687 & 0.652 & 0.566 & 0.5 \\
\hline
\end{tabular}

Table I: Acceleration dependence of $\pi_{*}$ and $\tau_{*}$

In Fig. 1 (a) we plot the three-tangle-dependence of $\pi$-tangle when $a=0,2 \omega c, 5 \omega c$, and $10 \omega c$. As expected from a fact that these are two different tripartite entanglement measures, 

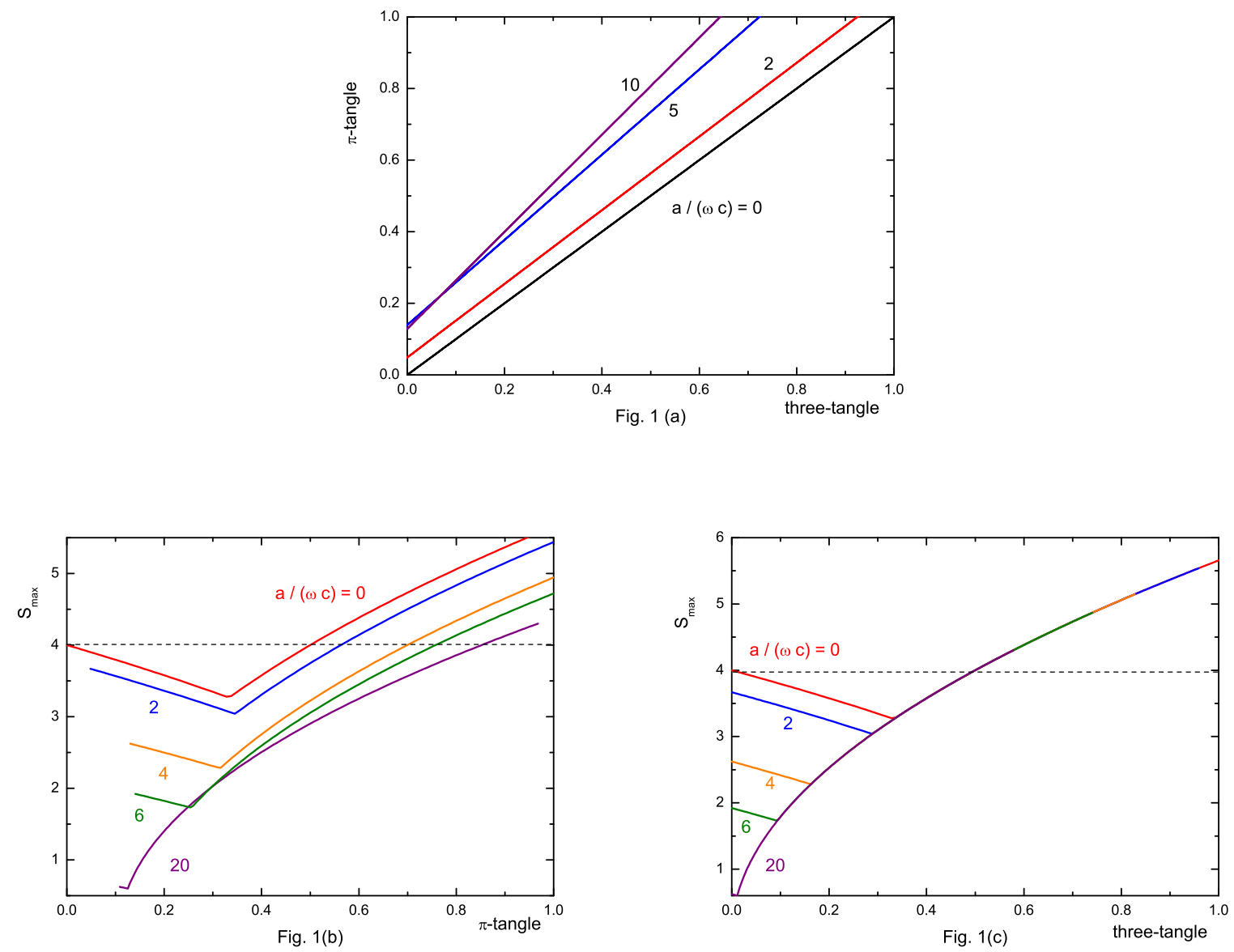

FIG. 1: (Color online) In (a) we plot the $\pi$-tangle (9) versus three-tangle (14). The $\pi$-tangle exhibits monotonous behavior with respect to the three-tangle. This fact is plausible because these tangles are two different measures for tripartite entanglement. In (b) and (c) we plot the tripartite entanglement-dependence of $S_{\max }$. These figures show that $S_{\max }$ exhibits a decreasing behavior in the small entanglement region. This fact seems to imply that entanglement is not unique physical resource for quantum mechanical non-locality.

$\pi$-tangle is monotonous with respect to three-tangle. Fig. 1(a) also shows that regardless of acceleration $a \pi$-tangle is larger than three-tangle, which was conjectured in Ref.[15, 26].

Fig. 1(b) and Fig. 1(c) show the tripartite entanglement-dependence of $S_{\max }$. As Fig. 1(b) exhibits, the violation of the Svetlichny inequality, i.e. $S_{\max }>4$, occurs when $\pi_{A B I}>$ $\pi_{*}$, where $\pi_{*}$ increases with increasing $a$. The critical value $\pi_{*}$ is given in Table I for various $a$. As Table I shows, $\pi_{*}$ approaches 1 at $a \rightarrow \infty$ limit, which implies that there is no violation 
of the Svetlichny inequality in this limit. Fig. 1(c) is a plot for the $\tau_{A B I}$-dependence of $S_{\max }$ for various $a$. As Fig. 1(c) exhibits, the violation of the Svetlichny inequality occurs when $\tau_{A B I}>0.5$ for all $a$. The maximum of the three-tangle, i.e. $\tau_{*}$, is dependent on Charlie's acceleration $a$. As Table I shows, $\tau_{*}$ exhibits a decreasing behavior with increasing $a$, and eventually approaches 0.5 in $a \rightarrow \infty$ limit. This fact also indicates that the state shared initially by Alice, Bob, and Charlie cannot have non-local property in the infinite Charlie's acceleration although it has nonzero tripartite entanglement.

If Alice, Bob, and Charlie share initially the MS state $\left|\psi_{s}\right\rangle_{A B C}$, Charlie's acceleration changes $\left|\psi_{s}\right\rangle_{A B C}$ into

$$
\begin{aligned}
\sigma_{A B I}=\frac{1}{2}\left[\begin{array}{c}
\cos ^{2} r|000\rangle \\
\end{array}\right. & \left\langle 000\left|+\sin ^{2} r\right| 001\right\rangle\left\langle 001\left|+\cos ^{2} \theta_{3} \cos ^{2} r\right| 110\right\rangle\langle 110| \\
& +\left(\sin ^{2} \theta_{3}+\cos ^{2} \theta_{3} \sin ^{2} r\right)|111\rangle\langle 111| \\
& +\cos \theta_{3} \cos ^{2} r\{|000\rangle\langle 110|+| 110\rangle\langle 000|\}+\sin \theta_{3} \cos r\{|000\rangle\langle 111|+| 111\rangle\langle 000|\} \\
& \left.+\cos \theta_{3} \sin ^{2} r\{|001\rangle\langle 111|+| 111\rangle\langle 001|\}+\sin \theta_{3} \cos \theta_{3} \cos r\{|110\rangle\langle 111|+| 111\rangle\langle 110|\}\right] .
\end{aligned}
$$

The maximum of $\langle S\rangle=\operatorname{tr}\left[\sigma_{A B I} S\right]$ was explicitly computed in Ref.[14], which has a form

$$
S_{\text {max }}=4\left[\cos ^{2} \theta_{3} \cos ^{2} 2 r+2 \sin ^{2} \theta_{3} \cos ^{2} r\right]^{1 / 2} \text {. }
$$

Thus, $S_{\max } \geq 4$ for $a=0$ and $S_{\max } \leq 4$ for $a=\infty$.

The $\pi$-tangle for $\sigma_{A B I}$ can be computed straightforwardly and its final expression is

$$
\pi_{M S}=\frac{1}{3}\left[\sin ^{2} \theta_{3}\left(2+\cos ^{2} r\right)+\sin ^{2} r \cos ^{2} r\left(1+\cos ^{2} \theta_{3}\right)^{2}\right] .
$$

In order to compute the three-tangle for $\sigma_{A B I}$ we express $\sigma_{A B I}$ in terms of eigenvectors as following:

$$
\sigma_{A B I}=\Lambda_{+}\left|\Psi_{+}\right\rangle\left\langle\Psi_{+}\left|+\Lambda_{-}\right| \Psi_{-}\right\rangle\left\langle\Psi_{-}\right|
$$

where

$$
\begin{aligned}
& \Lambda_{ \pm}=\frac{1 \pm \sqrt{\Delta}}{2} \\
& \left|\Psi_{ \pm}\right\rangle=\frac{1}{\mathcal{N}_{ \pm}}\left[X_{ \pm}|000\rangle+Y_{ \pm}|001\rangle+Z_{ \pm}|110\rangle+W_{ \pm}|111\rangle\right]
\end{aligned}
$$

In Eq. (20) $\Delta=\cos ^{2} \theta_{3}+\cos ^{2} r\left[\sin ^{2} \theta_{3}-\sin ^{2} r\left(1+\cos ^{2} \theta_{3}\right)^{2}\right]$ and

$$
\begin{array}{ll}
X_{ \pm}=\cos r(\mu \pm \sqrt{\Delta}) & Y_{+}=Y_{-}=\sin \theta_{3} \cos \theta_{3} \sin ^{2} r \\
Z_{ \pm}=\cos \theta_{3} X_{ \pm} & W_{ \pm}=\sin \theta_{3}\left(\cos ^{2} r \pm \sqrt{\Delta}\right)
\end{array}
$$


with $\mu=\cos ^{2} r-\sin ^{2} r \cos ^{2} \theta_{3}$. The normalization constants $\mathcal{N}_{ \pm}$are

$$
\begin{aligned}
\mathcal{N}_{ \pm}^{2} & =X_{ \pm}^{2}+Y_{ \pm}^{2}+Z_{ \pm}^{2}+W_{ \pm}^{2} \\
& = \pm 2 \sqrt{\Delta}\left[(1+\mu)\left(\cos ^{2} r \pm \sqrt{\Delta}\right)-\sin ^{2} r \cos ^{2} r \cos ^{2} \theta_{3}\left(1+\cos ^{2} \theta_{3}\right)\right]
\end{aligned}
$$

Then, it is easy to show $\left\langle\Psi_{+} \mid \Psi_{-}\right\rangle=0$. Now, we define

$$
\left|\Phi_{ \pm}(\varphi)\right\rangle=\sqrt{\Lambda_{+}}\left|\Psi_{+}\right\rangle \pm e^{i \varphi} \sqrt{\Lambda_{-}}\left|\Psi_{-}\right\rangle
$$

Then, $\sigma_{A B I}$ can be written as

$$
\sigma_{A B I}=\frac{1}{2}\left|\Phi_{+}(\varphi)\right\rangle\left\langle\Phi_{+}(\varphi)\left|+\frac{1}{2}\right| \Phi_{-}(\varphi)\right\rangle\left\langle\Phi_{-}(\varphi)\right| .
$$

The three-tangle $\tau\left(\Phi_{ \pm}\right)$for $\left|\Phi_{ \pm}(\varphi)\right\rangle$ are

$$
\tau\left(\Phi_{ \pm}\right)=4\left|\tilde{X}_{ \pm} \tilde{W}_{ \pm}-\tilde{Y}_{ \pm} \tilde{Z}_{ \pm}\right|^{2}
$$

where $\tilde{G}_{ \pm}=\sqrt{\Lambda_{+}} G_{+} / \mathcal{N}_{+} \pm e^{i \varphi} \sqrt{\Lambda_{-}} G_{-} / \mathcal{N}_{-}$with $G=X, Y, Z$, or $W$. If, thus, Eq. (24) is an optimal decomposition for $\sigma_{A B I}$, the three-tangle becomes

$$
\begin{aligned}
\tau\left(\sigma_{A B I}\right)= & \frac{4 \Lambda_{+}^{2}}{\mathcal{N}_{+}^{4}}\left(X_{+} W_{+}-Y_{+} Z_{+}\right)^{2}+\frac{4 \Lambda_{-}^{2}}{\mathcal{N}_{-}^{4}}\left(X_{-} W_{-}-Y_{-} Z_{-}\right)^{2} \\
& +\frac{4 \Lambda_{+} \Lambda_{-}}{\mathcal{N}_{+}^{2} \mathcal{N}_{-}^{2}}\left\{\left(X_{+} W_{-}+X_{-} W_{+}\right)-\left(Y_{+} Z_{-}+Y_{-} Z_{+}\right)\right\}^{2} \\
& +\frac{8 \Lambda_{+} \Lambda_{-}}{\mathcal{N}_{+}^{2} \mathcal{N}_{-}^{2}}\left(X_{+} W_{+}-Y_{+} Z_{+}\right)\left(X_{-} W_{-}-Y_{-} Z_{-}\right) \cos 2 \varphi
\end{aligned}
$$

Since $\left(X_{+} W_{+}-Y_{+} Z_{+}\right)\left(X_{-} W_{-}-Y_{-} Z_{-}\right)=\cos ^{2} r \sin ^{4} r \cos ^{4} \theta_{3} \sin ^{6} \theta_{3} \geq 0$, we have to choose $\varphi=\pi / 2$ to minimize $\tau\left(\sigma_{A B I}\right)$. Then, $\tau\left(\sigma_{A B I}\right)$ simply reduces to

$$
\tau\left(\sigma_{A B I}\right)=\cos ^{2} r \sin ^{2} \theta_{3}
$$

It is interesting to note that the three-tangle is much simpler than the $\pi$-tangle. From Eq. (17) and Eq. (27) one can derive the three-tangle-dependence of $S_{\max }$, which is

$$
S_{\max }=4 \sqrt{\cos ^{2} 2 r+\left(5-4 \cos ^{2} r-\tan ^{2} r\right) \tau\left(\sigma_{A B I}\right)}
$$

When $a=0$, Eq. (28) reduces to $S_{\max }=4 \sqrt{1+\tau\left(\sigma_{A B I}\right)}$. Thus, the violation of the Svetlichny inequality occurs for all nonzero three-tangle. When $a=\infty$, Eq. (28) reduces to $S_{\max }=4 \sqrt{2 \tau\left(\sigma_{A B I}\right)}$, which implies that the violation of the Svetlichny inequality occurs when $\tau\left(\sigma_{A B I}\right)>1 / 2$. 

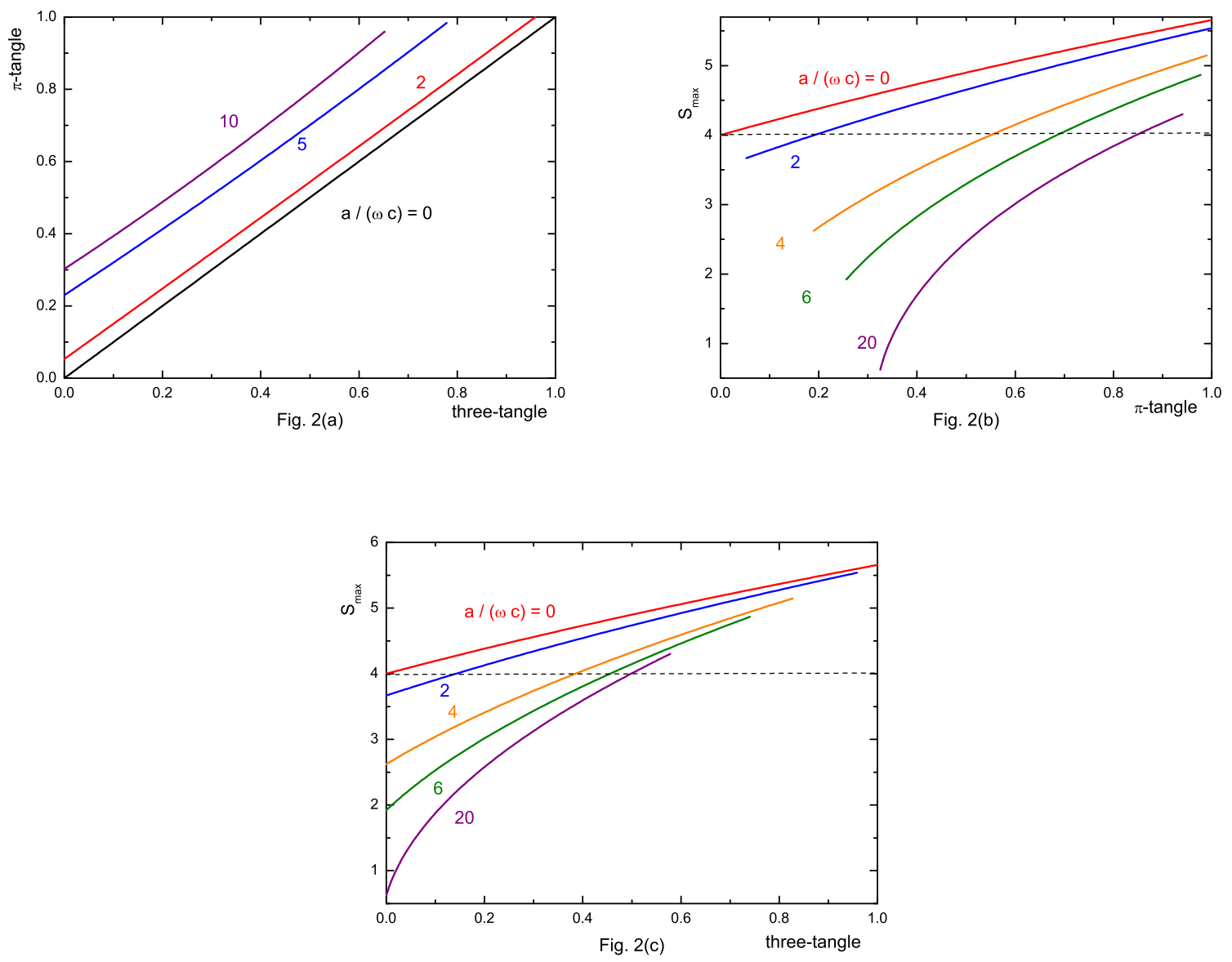

FIG. 2: (Color online) In (a) we plot the $\pi$-tangle (18) versus three-tangle (27). As Fig. 1(a) the $\pi$-tangle exhibits monotonous behavior with respect to the three-tangle. Regardless of acceleration $a$ the $\pi$-tangle is larger than the three-tangle, which might be true generally as conjectured in Ref.[15, 26]. In (b) and (c) we plot the tripartite entanglement-dependence of $S_{\max }$. Unlike Fig. 1(b) and Fig. 1(c) the decreasing behavior of $S_{\max }$ in small entanglement region disappears.

In Fig. 2(a) we plot the three-tangle-dependence of $\pi$-tangle for $\sigma_{A B I}$ when $a=0,2 \omega c$, $5 \omega c$, and $10 \omega c$. Like Fig. 1(a) the $\pi$-tangle (18) is monotonous with respect to the threetangle (27). Fig. 2(a) also indicates that $\pi$-tangle is in general larger than three-tangle. In Fig. 2(b) and Fig. 2(c) we plot the tripartite entanglement-dependence of $S_{\text {max }}$. Unlike Fig. 1(b) and Fig. 1(c) there is no decreasing behavior of $S_{\max }$ in these figures. From Fig. 2(b) and Fig. 2(c) we know that $\pi_{c}$ and $\tau_{c}$ increase with increasing $a$ if the violation of the Svetlichny inequality occurs when $\pi_{M S}>\pi_{c}$ and $\tau\left(\sigma_{A B I}\right)>\tau_{c}$. These critical values are 
given in Table II for various $a$. Table II shows that $\pi_{c} \rightarrow 1$ and $\tau_{c} \rightarrow 0.5$ in the infinite acceleration limit.

\begin{tabular}{c||c|c|c|c|c|c|c}
\hline$a / \omega c$ & 0 & 2 & 4 & 6 & 8 & 10 & 100 \\
\hline \hline$\pi_{c}$ & 0 & 0.191 & 0.250 & 0.685 & 0.746 & 0.780 & 0.901 \\
\hline$\tau_{c}$ & 0 & 0.142 & 0.385 & 0.456 & 0.479 & 0.488 & 0.5 \\
\hline
\end{tabular}

Table II: Acceleration dependence of $\pi_{c}$ and $\tau_{c}$

If Bob moves, instead of Charlie, with an uniform acceleration, the initial state $|\psi\rangle_{A B C}$ is transformed into

$$
\begin{aligned}
& \sigma_{A I C}=\frac{1}{2}\left[\cos ^{2} r|000\rangle\left\langle 000\left|+\sin ^{2} r\right| 010\right\rangle\left\langle 010\left|+\cos ^{2} \theta_{3}\right| 110\right\rangle\left\langle 110\left|+\sin ^{2} \theta_{3}\right| 111\right\rangle\langle 111|\right. \\
& +\cos r \cos \theta_{3}\{|000\rangle\langle 110|+| 110\rangle\langle 000|\}+\cos r \sin \theta_{3}\{|000\rangle\langle 111|+| 111\rangle\langle 000|\} \\
& \left.+\sin \theta_{3} \cos \theta_{3}\{|110\rangle\langle 111|+| 111\rangle\langle 110|\}\right] \text {. }
\end{aligned}
$$

The maximum of $\langle S\rangle=\operatorname{tr}\left[\sigma_{A I C} S\right]$ was given in Ref. [14], which is

$$
S_{\max }=4 \cos r\left[\cos ^{2} \theta_{3}+2 \sin ^{2} \theta_{3}\right]^{1 / 2} .
$$

The $\pi$-tangle for $\sigma_{A I C}$ can be straightforwardly computed and the final expression is

$$
\tilde{\pi}_{M S}=\frac{1}{3}\left[1+\sin ^{2} \theta_{3}-\cos ^{2} r \cos 2 \theta_{3}+\sin ^{2} r \cos 2 r+\sin ^{2} r \sqrt{\sin ^{4} r+4 \cos ^{2} r \cos ^{2} \theta_{3}}\right] .
$$

By similar method one can compute the three-tangle for $\sigma_{A I C}$, which is exactly the same with $\tau\left(\sigma_{A B I}\right)$ given in Eq. (27). Therefore, the three-tangle-dependence of $S_{\max }$ in this case is

$$
S_{\max }=4 \sqrt{\cos ^{2} r+\tau\left(\sigma_{A I C}\right)}
$$

Eq. (32) implies that the violation of the Svetlichny inequality arises for all nonzero $\tau\left(\sigma_{A I C}\right)$ when $a=0$. It also implies that $\tau\left(\sigma_{A I C}\right) \leq 1 / 2$ when $a \rightarrow \infty$ limit because $S_{\text {max }} \leq 4$ in this limit.

In this paper we have examined the tripartite entanglement-dependence of $S_{\max }=$ $\max \langle S\rangle$, where $S$ is the Svetlichny operator, when one party moves with an uniform acceleration $a$ with respect to other parties. If the initial tripartite state is the generalized GHZ state $\left|\psi_{g}\right\rangle_{A B C}$, the three-tangle-dependence of $S_{\max }$ is analytically derived in Eq. (15). 
As Fig. 1 shows, $S_{\max }$ exhibits a decreasing behavior in the small tripartite entanglement region while it exhibits a increasing behavior in the large tripartite entanglement region. This fact seems to suggest that the tripartite entanglement is not the only physical resource for the tripartite non-locality. If initial state is the MS state $\left|\psi_{s}\right\rangle_{A B C}$, the explicit relations between $S_{\max }$ and three-tangle are derived in Eq. (28) and Eq. (32). In this case the decreasing behavior of $S_{\max }$ disappears as Fig. 2 shows. The $a$-dependence of the critical values $\pi_{*}, \tau_{*}, \pi_{c}$, and $\tau_{c}$ is summarized in Table I and Table II.

It seems to be interesting to generalize our results to the tripartite bosonic cases [34]. In this case, however, it is highly difficult to compute $S_{\max }$ in non-inertial frame because the acceleration of one party transforms the qubit system at $a=0$ into a qudit system for nonzero $a$. In order to analyze this issue we should define the Svetlichny-like inequality in the qudit system.

As Eq. (18), Eq. (17), and Eq. (30) show, the violation of the Svetlichny inequality does not occur in $a \rightarrow \infty$ limit[36] even if the tripartite entanglement does not completely vanish in this limit. This fact suggests that although there is some connection between the tripartite non-locality and the tripartite entanglement, the entanglement is not unique resource for the non-locality. Then, what are other physical resources, which are responsible for the non-locality of quantum mechanics? As far as we know, we do not have definite answer so far. We will keep on studying this issue in the future.

Acknowledgement: This work was supported by the Kyungnam University Foundation Grant, 2011.

[1] A. Einstein, B. Podolsky and N. Rosen, Can quantum-mechanical description of physical reality be considered complete?, Phys. Rev. 47 (1935) 777.

[2] J. S. Bell, On the Einstein-Podolsky-Rosen Paradox, Physics, 1 (1964) 195.

[3] N. Gisin, Bell's inequality holds for all non-product states, Phys. Lett. A 154 (1991) 201.

[4] J. F. Clauser, M. A. Horne, A. Shimony and R. A. Holt, Proposed experiment to test local hidden-variable theories, Phys. Rev. Lett. 23 (1969) 880.

[5] A. K. Ekert, Quantum cryptography based on Bells theorem, Phys. Rev. Lett. 67 (1991) 661.

[6] S. Ghose, N. Sinclair, S. Debnath, P. Rungta, and R. Stock, Tripartite Entanglement versus 
Tripartite Nonlocality in Three-Qubit Greenberger-Horne-Zeilinger-Class States, Phys. Rev. Lett. 102 (2009) 250404.

[7] G. Svetlichny, Distinguishing three-body from two-body nonseparability by a Bell-type inequality, Phys. Rev. D 35 (1987) 3066.

[8] V. Coffman, J. Kundu and W. K. Wootters, Distributed entanglement, Phys. Rev. A 61 (2000) 052306.

[9] W. Dür, G. Vidal, and J. I. Cirac, Three qubits can be entangled in two inequivalent ways, Phys. Rev. A 62 (2000) 062314.

[10] H. A. Carteret and A. Sudbery, Local symmetry properties of pure three-qubit states, J. Phys. A 33 (2000) 4981.

[11] A. Ajoy and P. Rungta, Svetlichny's inequlity and genuine tripartite nonlocality in three-qubit pure states, Phys. Rev. A 81 (2010) 052334.

[12] W. G. Unruh, Notes on black-hole evaporation, Phys. Rev. D 14 (1976) 870.

[13] N. D. Birrel and P. C. W. Davies, Quantum Fields in Curved Space (Cambridge University Press, Cambridge, England, 1982).

[14] A. Smith and R. B. Mann, Persistence of Tripartite Nonlocality for Non-inertial observers, arXiv:1107.4633 [quant-ph].

[15] Y. U. Ou and H. Fan, Monogamy inequality in terms of negativity for three-qubit states, Phys. Rev. A 75 (2007) 062308.

[16] A. Cayley, On the Theory of Linear Transformations, Cambridge Math. J. 4 (1845) 193.

[17] A. Miyake, Classification of multipartite entangled states by multidimensional determinants, Phys. Rev. A 67 (2003) 012108.

[18] F. Verstraete, J. Dehaene and B. D. Moor, Normal forms and entanglement measures for multipartite quantum states, Phys. Rev. A 68 (2003) 012103.

[19] M. S. Leifer, N. Linden and A. Winter, Measuring polynomial invariants of multiparty quantum states, Phys. Rev. A 69 (2004) 052304.

[20] C. H. Bennett, D. P. DiVincenzo, J. A. Smokin and W. K. Wootters, Mixed-state entanglement and quantum error correction, Phys. Rev. A 54 (1996) 3824.

[21] A. Uhlmann, Fidelity and concurrence of conjugate states, Phys. Rev. A 62 (2000) 032307.

[22] R. Lohmayer, A. Osterloh, J. Siewert and A. Uhlmann, Entangled Three-Qubit States without Concurrence and Three-Tangle, Phys. Rev. Lett. 97 (2006) 260502. 
[23] C. Eltschka, A. Osterloh, J. Siewert and A. Uhlmann, Three-tangle for mixtures of generalized GHZ and generalized W states, New J. Phys. 10 (2008) 043014.

[24] E. Jung, M. R. Hwang, D. K. Park and J. W. Son, Three-tangle for Rank-3 Mixed States: Mixture of Greenberger-Horne-Zeilinger, $W$ and flipped $W$ states, Phys. Rev. A 79 (2009) 024306.

[25] E. Jung, D. K. Park, and J. W. Son, Three-tangle does not properly quantify tripartite entanglement for Greenberger-Horne-Zeilinger-type state, Phys. Rev. A 80 (2009) 010301(R).

[26] E. Jung, M. R. Hwang, D. K. Park, and S. Tamaryan, Three-Party Entanglement in Tripartite Teleportation Scheme through Noisy Channels, Quant. Inf. Comp. 10 (2010) 0377.

[27] G. J. He, X. H. Wang, S. M. Fei, H. X. Sun, and Q. Y. Wen, Three-tangle for high-rank mixed states, Commun. Theor. Phys. 55 (2011) 251.

[28] P. M. Alsing, I. Fuentes-Schuller, R. B. Mann, and T. E. Tessier, Entanglement of Dirac fields in noninertial frames, Phys. Rev. A 74 (2006) 032326.

[29] S. Weinberg, The quantum Theory of Fields I (Cambridge University Press, Cambridge, 1995).

[30] P. Caban, K. Podlaski, J. Rembieliński, A. Smoliński, and Z. Walczak, Entanglement and tensor product decomposition for two fermions, J, Phys, A: Math. Gen. 38 (2005) L79.

[31] K. Brádler, On two misconceptions in current relativistic quantum information arXiv:1108.5553 (quant-ph)].

[32] K. Brádler and R. Jáuregui, Comment on "Fermionic entanglement ambiguity in noninertial frames", Phys. Rev. A 85 (2012) 016301.

[33] M. Montero and E. Martín-Martínez, Reply to "Comment on 'Fermionic entanglement ambiguity in noninertial frames'", Phys Rev. A 85 (2012) 016302.

[34] M. R. Hwang, D. K. Park, and E. Jung, Tripartite entanglement in a noninertial frame, Phys. Rev. A 83 (2011) 012111.

[35] I. Fuentes-Schuller and R. B. Mann, Alice Falls into a Black Hole: Entanglement in Noninertial Frames, Phys. Rev. Lett. 95 (2005) 120404.

[36] N. Friis, P. Köhler, E. Martín-Martínez, and R. A. Bertlmann, Residual entanglement of accelerated fermions is not nonlocal, Phys. Rev. A 84 (2011) 062111. 